\title{
Association analysis of HSP90B1 with bipolar disorder
}

\author{
Chihiro Kakiuchi $\cdot$ Mizuho Ishiwata $\cdot$ Shinichiro Nanko $\cdot$ Hiroshi Kunugi \\ Yoshio Minabe · Kazuhiko Nakamura · Norio Mori · Kumiko Fujii · \\ Tadashi Umekage $\cdot$ Mamoru Tochigi $\cdot$ Kazuhisa Kohda $\cdot$ Tsukasa Sasaki · \\ Kazuo Yamada · Takeo Yoshikawa · Tadafumi Kato
}

Received: 24 May 2007/ Accepted: 24 July 2007 / Published online: 4 September 2007

(C) The Japan Society of Human Genetics and Springer 2007

\begin{abstract}
Pathophysiological role of endoplasmic reticulum (ER) stress response signaling has been suggested for bipolar disorder. The goal of this study was to test the genetic association between bipolar disorder and an ER chaperone gene, HSP90B1 (GRP94/gp96), which is located on a candidate locus, 12q23.3. We tested the genetic association between bipolar disorder and HSP90Bl by case-control studies in two independent Japanese sample sets and by a transmission disequilibrium test (TDT) in NIMH Genetics initiative bipolar trio samples (NIMH trios). We also performed gene expression analysis of HSP90B1 in lymphoblastoid cells. Among the 11 SNPs tested, rs17034977 showed significant association in both
\end{abstract}

C. Kakiuchi $\cdot$ M. Ishiwata $\cdot$ M. Tochigi $\cdot$ T. Kato $(\square)$

Laboratory for Molecular Dynamics of Mental Disorders,

RIKEN Brain Science Institute, 2-1 Hirosawa,

Wako, Saitama 351-0198, Japan

e-mail: kato@brain.riken.jp

\section{S. Nanko}

Department of Psychiatry and Genome Research Center,

Teikyo University School of Medicine, Tokyo, Japan

\section{H. Kunugi}

Department of Mental Disorder Research,

National Institute of Neuroscience,

National Center of Neurology and Psychiatry,

Tokyo, Japan

\section{Y. Minabe}

Department of Psychiatry and Neurobiology,

Kanazawa University Graduate School of Medical Science,

Kanazawa, Japan

K. Nakamura · N. Mori

Department of Psychiatry,

Hamamatsu University School of Medicine,

Hamamatsu, Japan
Japanese sample sets. The frequency of the SNP was lower in NIMH samples than in Japanese samples and there was no significant association in NIMH trios. Gene expression analysis of $H S P 90 B 1$ in lymphoblastoid cells suggested a possible relationship between the associated SNP and mRNA levels. HSP90B1 may have a pathophysiological role in bipolar disorder in the Japanese population, though further study will be needed to understand the underlying functional mechanisms.

Keywords Bipolar disorder - HSP90B1/GRP94/gp96 . Association study · Evi12 - Endoplasmic reticulum stress . Retrovirus

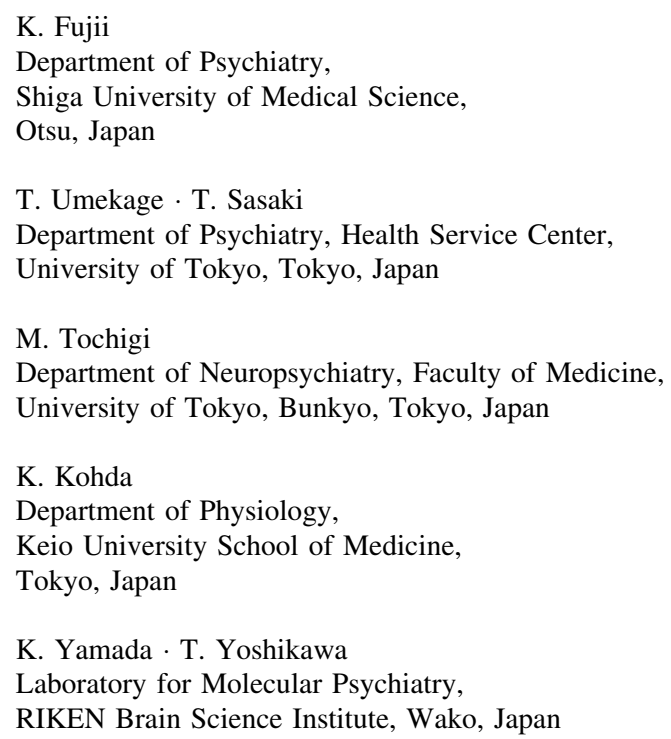




\section{Introduction}

Although the contribution of genetic factors to bipolar disorder has been evidenced by family, twin and adoption studies, the genetic risk factors for the illness are still controversial (Kato 2007). We previously suggested that endoplasmic reticulum (ER) stress response signaling is one of the candidate cascades that could be related to pathology of the illness (Kakiuchi et al 2003, 2005, 2006).

$\mathrm{ER}$ is a protein folding system. When unfolded proteins accumulate in ER, which could occur for a number of reasons, the ER stress response begins. ER stress response consists of four signaling cascades: (1) induction of ER chaperone such as HSPA5 (also named as GRP78 or BiP), HSP90B1 (GRP94 or gp96), CALR (Calreticulin), etc., which promotes the folding of unfolded proteins, (2) inhibition of protein synthesis, (3) induction of an ERassociated degradation pathway, which promotes the processing of unfolded proteins, and (4) induction of apoptosis when this system cannot process the unfolded proteins (Schroder and Kaufman 2005; Yoshida 2004).

When ER chaperones are consumed to fold unfolded proteins, HSPA5 is dissociated from ATF6 protein on the ER membrane, which causes cleavage of ATF6. Cleaved ATF6 protein induces the expression of ER chaperones and $X B P 1$. In parallel, dissociation of HSPA5 from IRE1 protein on the ER membrane causes dimerization of IRE1, which splices XBPI mRNA. The spliced XBPI mRNA encodes an active form of XBP1 that strongly induces the expression of target genes such as ER chaperones (Yoshida 2004).

Several lines of evidence have suggested that ER stress related genes are involved in the pathophysiology of affective illness including bipolar disorder. The first evidence was detection of ER chaperones such as HSPA5, HSP90B1 and CALR as target genes of a mood stabilizer, valproate, using differential display (Wang et al. 1999). Subsequently, increased expression of ER chaperones in the temporal cortex of depressed subjects who died by suicide was reported (Bown et al. 2000). One microarray study using postmortem brain samples showed altered expression of HSP90Bl ( $g$ p96) in the bipolar disorder patients (Jurata et al. 2004). The anti-malarial drug mefloquine, which is known to cause psychiatric symptoms including bipolar disorder in susceptible individuals (Even et al. 2001), is reported to induce ER stress in rat neurons (Dow et al. 2003, 2005). Methamphetamine (MAP), a psychostimulant causing a manic state, is known to induce ER chaperone genes in the mouse brain (Jayanthi et al. 2004). Foot shock stress or increase of serotonergic neurotransmission by the treatment with 5-hydroxy-1tryptophan and clorgyline caused enhancement of XBP1 splicing in rats (Toda et al. 2006). Two common mood stabilizers, valproate and lithium, have neuroprotective effects by inducing ER chaperones (Hiroi et al. 2005; Kim et al. 2005; Wang et al. 2003). Valproate also enhanced the ER stress response to thapsigargin (Kakiuchi et al. 2003).

We previously showed by DNA microarray analysis that $X B P 1$ and HSPA5 are down-regulated in the lymphoblastoid (LB) cells of monozygotic twins with bipolar disorder compared with healthy co-twins (Kakiuchi et al. 2003). Induction of XBP1 and HSPA5 mRNA by thapsigargin was reduced in the patients' cell lines. Recently, So et al. (2007) also reported that induction of XBP1 and CHOP by thapsigargin and tunicamycin was attenuated in the lymphoblastoid cells derived from patients with bipolar disorder. Although we also reported that a functional polymorphism of XBP1 was associated with bipolar disorder, the genetic association was not replicated in Caucasian samples and Taiwanese samples (Cichon et al. 2004; Hou et al. 2004). We further performed a genetic association study of HSPA5 and demonstrated nominal association on rs16927997 in the Japanese population (Kakiuchi et al. 2005). However, the association was not replicated in an independent sample set in that study and no association was found in NIMH trio samples. The other ER stress related genes ATF4/ATF5 were not found to be associated with bipolar disorder (Kakiuchi et al. 2007). Thus, association of the ER stress related genes with bipolar disorder is still controversial.

In this study, we investigated a potential genetic association of $H S P 90 B 1$, the other ER chaperone gene, with bipolar disorder. HSP90Bl expression is induced by treatment with valproate and lithium (Shao et al. 2006) and its expression is altered in the postmortem brain of bipolar disorder patients (Bown et al. 2000). The HSP90B1 gene is located on $12 \mathrm{q} 23.3$. Many studies have shown the significant linkage of 12q23-24 with bipolar disorder (Curtis et al. 2003; Dawson et al. 1995; Detera-Wadleigh et al. 1999; Ekholm et al. 2003; Ewald et al. 1998; Green et al. 2005; Maziade et al. 2005; Morissette et al. 1999). We also performed expression analysis in LB cells derived from patients and controls.

\section{Materials and methods}

\section{Subjects}

For the case control study in the Japanese population, a total of 449 patients with bipolar disorder $(50.2 \pm 13.4$ years old, 216 males and 233 females, 318 with bipolar I disorder, 126 with bipolar II disorder and 5 with schizoaffective disorder, bipolar type) and 543 controls (43.4 \pm 15.0 years old, 269 males and 274 females) were analyzed. These samples are similar to those previously 
described and included two independently collected sample sets (Kakiuchi et al. 2005). One set consisted of samples that were not age- and sex-matched (MDMD samples), including 212 patients with bipolar disorder $(49.0 \pm 13.5$ years old, 87 males and 125 females, 152 with bipolar I and 60 with bipolar II disorder) and 304 controls (37.1 \pm 14.8 years old, 149 males and 155 females). The other set was age- and sex-matched samples ("MPS samples"), which included 237 patients with bipolar disorder (51.2 + 13.2 years old, 129 males and 108 females, 166 with bipolar I, 66 with bipolar II, and 5 with schizoaffective disorder bipolar type) and 239 controls (51.5 \pm 10.7 years old, 120 males and 119 females $)$. To test the specificity of the findings, 227 patients with schizophrenia $(46.0 \pm 14.9$ years old, 130 males and 97 females) were also examined. They were diagnosed according to the DSM-IV criteria (American Psychiatric Association) by the consensus of at least two psychiatrists. Structured interviews were not used for the diagnosis, except for use of a structured interview, the Mini-International Neuropsychiatric Interview (M.I.N.I.) (Sheehan et al. 1998), for recently recruited 17 patients. Controls were selected from students, nurses, office workers, and doctors in participating institutes, and their friends. A senior psychiatrist, one of authors, interviewed them and they did not have major mental disorders.

In Japanese, no significant population stratification has been repeatedly reported in several studies including a part of our samples (Arinami et al. 2005; Kakiuchi et al. 2003; Yamada et al. 2004). For the transmission disequilibrium test, we analyzed a total of 238 trio samples from NIMH Genetics Initiative Pedigrees. Only one trio was obtained from one family. The criteria, by which the trio was selected from a pedigree, were as follows: (1) DNA is available for parents and the proband, (2) if multiple complete trios were found in one pedigree, the trio with a younger generation was selected, and (3) if multiple trios were available in one generation, an elder sibling was selected as the proband. Data and biomaterials of the $\mathrm{NIMH}$ pedigrees were collected in four projects that participated in the NIMH Bipolar Disorder Genetics Initiative. From 1991 to 1998, the Principal Investigators and CoInvestigators were: Indiana University, Indianapolis, IN, U01 MH46282, J. Nurnberger, M. Miller, and E. Bowman; Washington University, St. Louis, MO, U01 MH46280, T. Reich, A. Goate, and J. Rice; Johns Hopkins University, Baltimore, MD U01 MH46274, J. R. DePaulo, Jr., S. Simpson, and C. Stine; NIMH Intramural Research Program, Clinical Neurogenetics Branch, Bethesda, MD, E. Gershon, D. Kazuba, and E. Maxwell. In these pedigrees, linkage with 12q23-q24 was reported (Detera-Wadleigh et al. 1999). For the mRNA expression analysis, we examined the mRNA expression level of HSP90B1 in LB cells derived from 23 patients $(51.3 \pm 11.9$ years old, 16 males and 7 females) and 19 controls (49.0 \pm 13.1 years old, 12 males and 7 females). For the Southern blotting analysis, we used genomic DNA derived from LB cells of 53 patients who were also included in the Japanese casecontrol analysis. Written informed consent was obtained from all subjects. The Ethics Committees of the Brain Science Institute (RIKEN) and participating institutes approved the study.

\section{Genetic association studies}

In total, seven SNPs (rs1165678, rs1165681, rs1165687, rs1882019, rs17034977, rs703657 and rs2293618) were selected according to the LD Map Database on SNPbrowser $^{\mathrm{TM}}$ of Applied Biosystems (Applied Biosystems; Foster city, CA, USA) (Fig. 1). These SNPs were selected because their TaqMan probes were commercially available

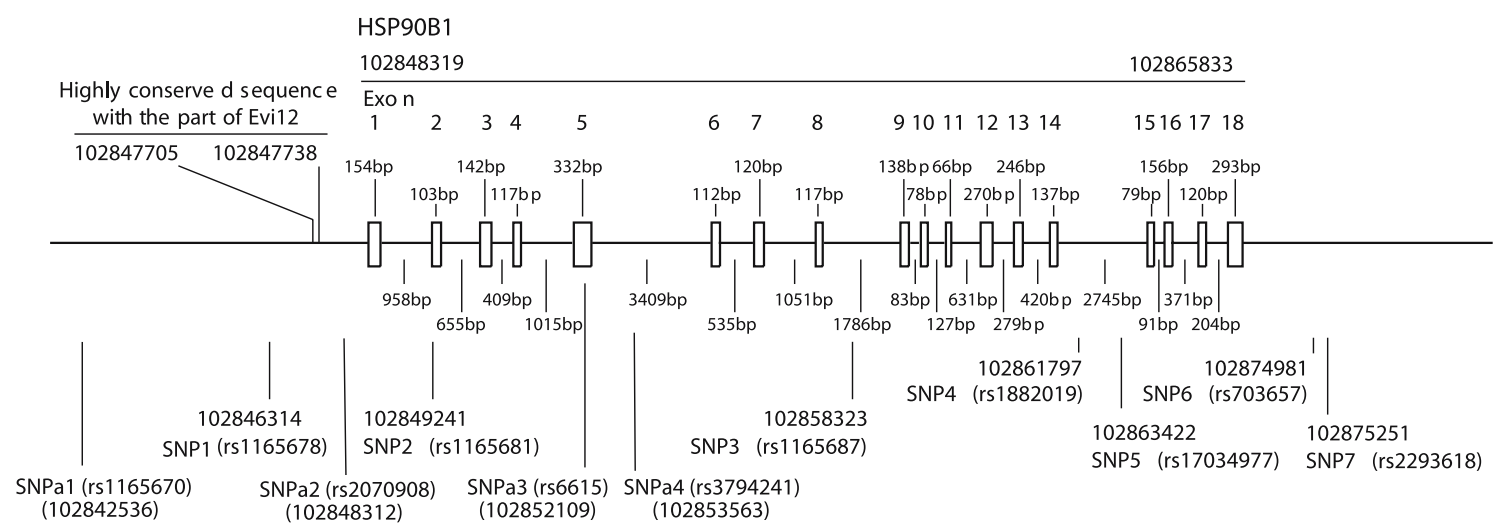

Fig. 1 The genomic structure of HSP90B1. HSP90B1 consists of 18 exons. A highly conserved 33-bp sequence is observed in the upstream region. Number indicates the position in the UCSC Human Genome Browser, March 2006 
and they are polymorphic in Japanese. Based on the results obtained, we genotyped 4 additional SNPs (rs1165670, rs2070908, rs6615 and rs3794241). They were selected from the database because they have similar frequency and are supposed to be linked with rs17034977. Genotyping was performed using commercially available TaqMan probes and $\mathrm{ABI} 7900 \mathrm{HT}$ according to the protocol recommended by the manufacturer (Applied Biosystems). Assessment of linkage disequilibrium (LD) patterns by the standardized disequilibrium coefficient $\left(D^{\prime}\right)$ and squared correlation coefficient $\left(r^{2}\right)$, analysis of haplotypic distribution and frequencies, and haplotype TDT analysis were performed using the COCAPHASE and TDTPHASE programs (http://www.portal.litbio.org/Registered/Option/ unphased.html).

\section{Sequencing}

To identify the possible functional polymorphisms that are linked to the haplotype associated with bipolar disorder, we sequenced the upstream region, $5^{\prime}$-untraslated region (UTR), exon14, exon17 and exon18, in which the existence of missense polymorphisms has been shown in UCSC Human Genome Browser, March 2006 (http://www. genome.ucsc.edu/), and $3^{\prime}$-UTR of HSP90B1 in 44 patients with bipolar disorder. We also sequenced around the highly conserved region with Evi12. Primer sequences are as follows: promotor region and $5^{\prime}$-UTR, forward 5'-CTGACCAATCGGAAGGAG and reverse 5'-ATCA CTCACCGAAGGTCA; exon14, forward 5'-GACAGTT GAAAGACAATTGCTC and reverse 5'-CGTAAACTTG TTGTCTACCT; exon17, forward 5'-GAAAGAATGCTT CGCCTCAG and reverse 5'-ACTCTGTCCTAACTTTG CCAGAAC; exon 18 and $3^{\prime}$-UTR, forward 5'-CATGCACA AACCTGTGAG and reverse 5'-TCAGCCAAGTATTA GGTAGT; around Evi12, forward 5'-GCACATATCTCT ACTGTAATCAGAACGAG and reverse 5'-CCTGGGG TTTCGTTGGAAGTG.

\section{Quantification of mRNA levels}

We examined the mRNA expression level of HSP90B1 in LB cells. We transformed lymphocytes from peripheral blood by Epstein-Barr virus and cultured LB cells in the medium of RPMI1640 (Sigma; Saint Louis, Missouri, USA) containing $10 \%$ fetal bovine serum (FBS) as the same methods described previously (Kato et al. 2003). Then, LB cells were collected into TRIZOL reagents (Invitrogen; San Diego, CA, USA). Total RNA was extracted and single strand cDNA was synthesized using oligo (dT) primer and SuperScript II reverse transcriptase (Invitrogen) by the same methods described previously (Kakiuchi et al. 2003). For the quantification of mRNA levels, real-time quantitative PCR using TaqMan chemistry was performed according to the manufacturer's protocol (Applied Biosystems). For quantitative PCR, ABI7900HT was used and the relative ratios to GAPDH were calculated. The experiments were performed in quadliplicate. Primer and probe mixture of $H S P 90 B 1$ and $G A P D H$ was obtained from assay-on-demand products (Applied Biosystems). $T$ test was used for comparison of the expression levels between controls and patients in LB cells.

\section{Southern blotting}

Southern blotting analysis was performed to examine the insertion/deletion mutations around the HSP9OBI gene. Ten micrograms of genomic DNA derived from LB cells was digested by EcoRV (Takara; Otsu, Shiga, Japan) and run on a $0.8 \%$ agarose gel. After transferring to nylon membrane, hybridization was performed using two kinds of digoxigenin-labeled probes. Probes were made as follows. PCR-products were cloned into pCR-2.1-TOPO Vector (Invitrogen), and then internally labeled with digoxigenin using a PCR DIG Probe Synthesis Kit (Roche, Germany) according to the manufacturer's protocol. Primers are as follows: forward, ATGGGACCTGACCC AAGT and reverse, GTAATGTCCCACACAATTTA GGAG, which detect 4642 bp fragments on the upstream region of HSP90B1 (chromosome 12, 102839756102844427, UCSC Human Genome Browser, March 2006 Assembly); forward, CACGAATCCTCATTGGGT and reverse, CAGTTAGATGATGTTGCTCTCT, which detect 6050 bp fragments (chromosome 12, 102844428102850477). Hybridization of the probes was detected by LAS-3000 luminoimage analyzer (Fujifilm; Tokyo, Japan) using a part of DIG High Prime DNA Labeling and Detection Starter Kit II (Roche).

Statistical analysis

For statistical analysis, Chi-square test and Mann-Whitney $U$ test were used. The standardized disequilibrium coefficient $\left(D^{\prime}\right)$ and squared correlation coefficient $\left(r^{2}\right)$ was calculated using the COCAPHASE program. The standardized disequilibrium coefficient $\left(D^{\prime}\right)$ and squared correlation coefficient $\left(r^{2}\right)$ in the trio samples was calculated using the TDTPHASE program. For the association analysis, $P$ values obtained by the Chi-square test before the Bonferroni correction were shown. 


\section{Results}

Case control study for bipolar disorder in Japanese samples

The structure of HSP9OB1 is shown in Fig. 1. The results of association analysis of single SNPs are shown in Table 1. rs17034977 was significantly associated with bipolar disorder in both sample sets, "MDMD samples" and "MPS samples", although they are no more significant after the Bonferroni correction. The allelic association of bipolar disorder with rs17034977 was statistically significant even after the Bonferroni correction in the combined samples $(P=0.005$ for genotype and $P=0.001$ for allele. After Bonferroni correction considering seven markers and two comparisons, $P=0.07$ and $P=0.014$, respectively). There were no significant relationship between lithium response and the rs17034977 genotypes in the patients whose clinical information on lithium response was available (data not shown).

Because only one SNP was associated with bipolar disorder in a case control study in Japanese, we genotyped four additional SNPs (rs1165670, rs2070908, rs6615 and rs3794241) which have similar frequency and are supposed to be linked with rs17034977. None of these SNPs (SNPs a1-a4) were significantly associated with bipolar disorder (Table 1). Linkage disequilibrium by the $D^{\prime}$ and $r^{2}$ in the control subjects is shown in Fig. 2. Because SNPs 3-7 are in the haploptype block, we performed the haplotype analysis of SNPs 3-7 (Table 2). There are three haplotypes, CAATT, CGAAC, and CGCTT, of which, CGCTT was significantly associated with bipolar disorder (global significance by random permutation test [10,000 times] using COCAPHASE program was $P=0.0026)$. This haplptype is determined by the SNP5, and thus SNP5 can be regarded as the tag SNP in this haplotype block.

Table 1 The result of case-control studies in Japanese population

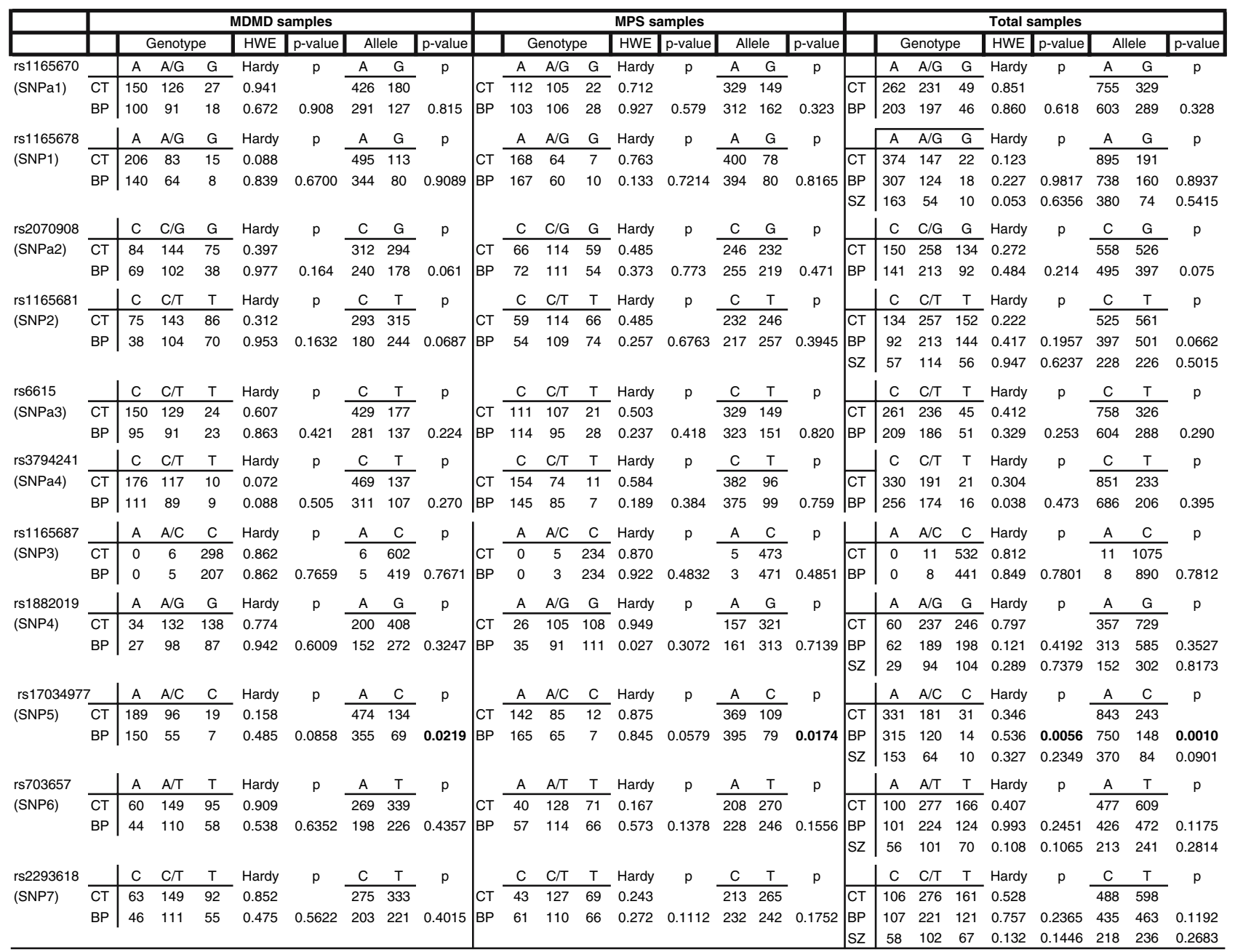

For the SNPs a1-a4, the number of subjects were 446 for bipolar disorder and 542 for controls. 


\begin{tabular}{|c|c|c|c|c|c|c|c|c|c|c|c|c|}
\hline & & $\begin{array}{c}D^{\prime} \\
\text { SNPa1 } \\
\text { rs } 1165670\end{array}$ & $\begin{array}{c}\text { SNP1 } \\
\text { rs1165678 }\end{array}$ & $\begin{array}{c}\text { SNPa2 } \\
\text { rs2070908 }\end{array}$ & $\begin{array}{c}\text { SNP2 } \\
\text { rs1165681 }\end{array}$ & $\begin{array}{l}\text { SNPa3 } \\
\text { rs } 6615\end{array}$ & $\begin{array}{c}\text { SNPa4 } \\
\text { rs3794241 }\end{array}$ & $\begin{array}{c}\text { SNP3 } \\
\text { rs1165687 }\end{array}$ & $\begin{array}{c}\text { SNP4 } \\
\text { rs1882019 }\end{array}$ & $\begin{array}{c}\text { SNP5 } \\
\text { rs17034977 }\end{array}$ & $\begin{array}{r}\text { SNP6 } \\
\text { rs703657 }\end{array}$ & $\begin{array}{c}\text { SNP7 } \\
\text { rs2293618 }\end{array}$ \\
\hline SNPa1 & rs1165670 & & 1 & 1 & 1 & 1 & 0.1005 & 1 & 0.6398 & 0.6569 & 0.68 & 0.6687 \\
\hline SNP1 & rs1165678 & 0.0932 & & 0.9849 & 1 & 0.9599 & 0.6009 & 0.8745 & 0.8724 & 1 & 0.8478 & 0.9485 \\
\hline SNPa2 & rs2070908 & 0.4623 & 0.1956 & & 1 & 1 & 0.2524 & 0.7385 & 0.6904 & 0.7107 & 0.1034 & 0.07843 \\
\hline SNP2 & rs1165681 & 0.464 & 0.2009 & 0.9963 & & 1 & 0.2554 & 0.7377 & 0.6892 & 0.7117 & 0.09883 & 0.07412 \\
\hline SNPa3 & rs 6615 & 0.1874 & 0.08476 & 0.4054 & 0.4039 & & 0.3821 & 1 & 0.8698 & 0.9051 & 0.8203 & 0.8272 \\
\hline SNPa4 & rs3794241 & 0.001204 & 0.02115 & 0.01645 & 0.01677 & 0.09293 & & 0.044 & 0.3828 & 0.3635 & 0.4606 & 0.4473 \\
\hline SNP3 & rs1165687 & 0.004467 & 0.03665 & 0.005271 & 0.00524 & 0.004409 & $7.25 \mathrm{E}-05$ & & 1 & 1 & 1 & 1 \\
\hline SNP4 & rs1882019 & 0.08686 & 0.07927 & 0.2188 & 0.2172 & 0.6682 & 0.0824 & 0.004992 & & 0.979 & 1 & 1 \\
\hline SNP5 & rs17034977 & 0.05433 & 0.0618 & 0.1548 & 0.1558 & \begin{tabular}{l|l}
0.1018 \\
\end{tabular} & 0.01045 & 0.002962 & 0.1349 & & 1 & 1 \\
\hline SNP6 & rs703657 & 0.2564 & 0.1956 & 0.008908 & 0.008173 & 0.2274 & 0.04566 & 0.008056 & 0.3827 & 0.2271 & & 1 \\
\hline SNP7 & rs2293618 & 0.238 & 0.235 & 0.005343 & 0.004789 & 0.241 & 0.04485 & 0.01252 & 0.3987 & 0.2366 & 0.9597 & \\
\hline
\end{tabular}

D' larger than 0.9 was shown in shaded cells.

Fig. 2 Intermarker linkage disequilibrium pattern in Japanese samples. The standardized disequilibrium coefficient $\left(D^{\prime}\right)$ and squared correlation coefficient $\left(r^{2}\right)$ was calculated for total controls using the COCAPHASE program. $D^{\prime}$ larger than 0.9 was shown in shaded cells

Table 2 Haplotype analysis of HSP90B1 in Japanese BP samples

\begin{tabular}{|c|c|c|c|c|c|c|c|c|c|}
\hline \multirow{2}{*}{$\frac{\text { Haplotype }}{(\text { SNPs3-7) }}$} & \multicolumn{3}{|c|}{ MDMD samples } & \multicolumn{3}{|c|}{ MPS samples } & \multicolumn{3}{|c|}{ Total samples } \\
\hline & BP & CT & $P$ & BP & CT & $P$ & BP & CT & $P$ \\
\hline 1. $\mathrm{C}-\mathrm{A}-\mathrm{A}-\mathrm{T}-\mathrm{T}$ & $150(0.36)$ & $197(0.33)$ & 0.27 & $158(0.34)$ & $155(0.33)$ & 0.72 & $308(0.35)$ & $352(0.33)$ & 0.31 \\
\hline 2. $\mathrm{C}-\mathrm{G}-\mathrm{A}-\mathrm{A}-\mathrm{C}$ & $196(0.47)$ & $266(0.44)$ & 0.35 & $224(0.48)$ & $205(0.43)$ & 0.15 & $420(0.48)$ & $471(0.44)$ & 0.094 \\
\hline \multirow[t]{2}{*}{ 3. C-G-C-T-T } & $66(0.16)$ & $133(0.22)$ & 0.012 & $78(0.16)$ & $106(0.22)$ & 0.027 & $144(0.16)$ & $239(0.22)$ & 0.00094 \\
\hline & \multicolumn{3}{|c|}{ Global $P=0.037$} & \multicolumn{3}{|c|}{ Global $P=0.071$} & \multicolumn{3}{|c|}{ Global $P=0.0026$} \\
\hline
\end{tabular}

Global significance was calculated by a random permutation test (10,000 times) using the COCAPHASE program

Only haplotypes that were verified at least once were analyzed

Significant $P$-values are shown in bold

TDT analysis for bipolar disorder in NIMH trios

We examined the association in other ethnicities by TDT analysis of NIMH bipolar trio samples, which are mainly derived from Caucasians. No significant over-transmission was observed in any SNPs (Table 3). rs17034977 was not significantly associated. Linkage disequilibrium by the $D^{\prime}$ and $r^{2}$ in the transmitted allele is shown in Fig. 3.

Case control study for schizophrenia

in Japanese samples

We further performed association analysis of the HSP90B1 locus for schizophrenia in Japanese samples because schizophrenia and bipolar disorder are known to share a common genetic background. We genotyped six of the SNPs (SNP1, 2, 4, 5, 6, and 7). The genotype and allele frequencies were compared with the total Japanese controls used for the analysis of bipolar disorder. No significant association was observed in single SNPs (Table 1). However, the result should be treated with caution, because there was a significant difference of gender between patients with schizophrenia and controls $(P<0.05)$.
HSP90B1 mRNA levels in lymphoblastoid cells

We examined the mRNA expression level of HSP90B1 in LB cells derived from 22 bipolar disorder patients and 19 controls. There were no statistically significant differences between controls and patients in relative expression levels of $H S P 90 B 1$ to $G A P D H$ in the cells $(P=0.468$, MannWhitney $U$ test; Fig. 4).

The carrier of C allele of SNP5 (rs17034977) had significantly lower expression levels of HSP90B1 $(n=25$, $0.029 \pm 0.008$, mean \pm SD) compared with those carrying homozygous A alleles $(n=17,0.036 \pm 0.009, P=0.035$, Mann-Whitney $U$ test; Fig. 5).

Search for possible functional polymorphisms

We tried to identify the functional SNPs linking to the haplotype responsible for the association with bipolar disorder and the alteration of HSP90B1 expression level. Public databases were searched and 44 DNA samples of bipolar disorder patients were sequenced. Putative functional polymorphisms, such as variation within the promoter region, polymorphisms altering amino acid 
Table 3 The result of TDT analysis in NIMH trio samples

\begin{tabular}{|c|c|c|c|c|}
\hline & Allele & Transmitted & Not-transmitted & $P$ value \\
\hline rs1165670 & A & $352(0.7395)$ & $342(0.7185)$ & 0.466 \\
\hline (SNPa1) & G & $124(0.2605)$ & $134(0.2815)$ & \\
\hline rs1165678 & A & $288(0.6025)$ & $293(0.613)$ & 0.741 \\
\hline (SNP1) & $\mathrm{G}$ & $190(0.3975)$ & $185(0.387)$ & \\
\hline rs2070908 & $\mathrm{C}$ & $318(0.6681)$ & $316(0.6639)$ & 0.891 \\
\hline (SNPa2) & $\mathrm{G}$ & $158(0.3319)$ & $160(0.3361)$ & \\
\hline rs1165681 & $\mathrm{C}$ & $159(0.3326)$ & $163(0.341)$ & 0.784 \\
\hline (SNP2) & $\mathrm{T}$ & $319(0.6674)$ & $315(0.659)$ & \\
\hline rs6615 & $\mathrm{C}$ & $416(0.8739)$ & $419(0.8803)$ & 0.767 \\
\hline (SNPa3) & $\mathrm{T}$ & $60(0.1261)$ & $57(0.1197)$ & \\
\hline rs3794241 & $\mathrm{C}$ & $422(0.8866)$ & $432(0.9076)$ & 0.286 \\
\hline (SNPa4) & $\mathrm{T}$ & $54(0.1134)$ & $44(0.09244)$ & \\
\hline rs1165687 & A & $231(0.4833)$ & $236(0.4937)$ & 0.746 \\
\hline (SNP3) & $\mathrm{C}$ & $247(0.5167)$ & $242(0.5063)$ & \\
\hline rs1882019 & A & $49(0.1025)$ & $49(0.1025)$ & 1.000 \\
\hline (SNP4) & G & $429(0.8975)$ & $429(0.8975)$ & \\
\hline rs 17034977 & A & $441(0.9226)$ & 437 (0.9142) & 0.636 \\
\hline (SNP5) & $\mathrm{C}$ & $37(0.07741)$ & $41(0.08577)$ & \\
\hline rs 703657 & A & $160(0.3347)$ & $152(0.318)$ & 0.581 \\
\hline (SNP6) & $\mathrm{T}$ & $318(0.6653)$ & $326(0.682)$ & \\
\hline rs2293618 & $\mathrm{C}$ & $391(0.818)$ & $387(0.8096)$ & 0.740 \\
\hline (SNP7) & $\mathrm{T}$ & $87(0.182)$ & $91(0.1904)$ & \\
\hline
\end{tabular}

Number (frequency)

sequence, and those in untranslated regions (UTR), were searched for. In the non-coding region immediately upstream of the HSP90B1 gene, there is a conserved region. This region corresponds to the common virus integration site Evi12, the target of Cas- $\mathrm{Br}-\mathrm{M}$ murine leukemia virus (Valk et al. 1999; van den Akker et al. 2005) (Fig. 1). This region was also sequenced. In the database of UCSC Human Genome Browser, March 2006 (http://www.genome.ucsc.edu/), a total of 13 SNPs were suggested to exist in the 500-bp upstream region (rs3216180, rs17034921, rs2722187, rs2070908), 5'-UTR (rs 17797090), 3'-UTR (rs1051185, rs11547719, rs2307842), or as missense polymorphisms in exon14 (rs3209749), exon17 (rs17034989, rs17159034, rs3037197) and exon18 (rs11547722) of HSP90B1. In the conserved region, there were two SNPs (rs17034917 and rs17034919).

In the 44 samples, rs2722187, rs17797090, rs17034989, rs17159034, rs11547722, rs1051185, rs11547719, rs2307842 and rs3209749 were not polymorphic. rs3216180 (mainly linked with haplotype 1 [CAATT]), rs17034921 (mainly linked with haplotype 1), rs2070908 (linked with rs1165681) and rs3037197 (mostly linked with rs1882019) were polymorphic but not linked to haplotype 3 [CGCTT]. rs17034917 was not polymorphic and rs17034919 was linked with rs17034921, but not linked with haplotype 3 .

To rule out a possibility of large deletion or insertion affecting transcriptional activity, the upstream region spanning about $10 \mathrm{~kb}$ of $H S P 90 B 1$ gene was examined by Southern blotting. We did not observe an extra band, suggesting the insertion or deletion in the DNA of 53 patients with bipolar disorder derived from lymphoblastoid cells (data not shown).

\section{Discussion}

In this study, we showed the association of a SNP within the HSP90B1 locus (SNP5, rs17034977) with bipolar disorder in a Japanese population. No significant association was observed in NIMH bipolar disorder trio samples and Japanese schizophrenia samples. We examined the mRNA expression levels of $H S P 90 B 1$. There was no significant difference between controls and patients. The $\mathrm{C}$ allele of SNP5 (rs17034977) was significantly associated with lower expression levels of HSP90B1.

Although we searched for functional SNPs responsible for the association, no SNPs linked with the associated haplotype was identified. We did not find any deletion or insertion affecting the restriction fragment length examined by the Southern blot analysis in the upstream region. There

\begin{tabular}{|c|c|c|c|c|c|c|c|c|c|c|c|c|}
\hline & & $\begin{array}{c}D^{\prime} \\
\text { SNPa1 } \\
\text { rs } 1165670\end{array}$ & $\begin{array}{c}\text { SNP1 } \\
\text { rs1165678 }\end{array}$ & $\begin{array}{c}\text { SNPa2 } \\
\text { rs } 2070908\end{array}$ & $\begin{array}{c}\text { SNP2 } \\
\text { rs1165681 }\end{array}$ & $\begin{array}{l}\text { SNPa3 } \\
\text { rs } 6615\end{array}$ & $\begin{array}{c}\text { SNPa4 } \\
\text { rs3794241 }\end{array}$ & $\begin{array}{c}\text { SNP3 } \\
\text { rs1165687 }\end{array}$ & $\begin{array}{c}\text { SNP4 } \\
\text { rs1882019 }\end{array}$ & $\begin{array}{c}\text { SNP5 } \\
\text { rs17034977 }\end{array}$ & $\begin{array}{r}\text { SNP6 } \\
\text { rs703657 }\end{array}$ & $\begin{array}{c}\text { SNP7 } \\
\text { rs2293618 }\end{array}$ \\
\hline SNPa1 & rs1165670 & & 1 & 0.9878 & 0.9878 & 0.9207 & $\mid 0.1477$ & 0.896 & 0.8171 & 0.5583 & 0.853 & 0.7113 \\
\hline SNP1 & rs1165678 & 0.23 & & 1 & 1 & 0.953 & 0.6657 & 0.7142 & 0.8879 & 0.5211 & 0.6995 & 0.7354 \\
\hline SNPa2 & rs2070908 & 0.6919 & 0.3243 & & 1 & 1 & 0.3726 & 0.8762 & 0.9257 & 0.6976 & 0.6073 & 0.05459 \\
\hline SNP2 & rs1165681 & 0.6853 & 0.3274 & 0.9906 & & 1 & 0.3766 & 0.8774 & 0.9263 & 0.6965 & 0.6096 & 0.05071 \\
\hline SNPa3 & rs 6615 & 0.04307 & 0.08551 & 0.07166 & 0.07234 & & 0.5894 & 0.6906 & 0.9533 & 1 & 0.8216 & 0.7325 \\
\hline SNPa4 & rs3794241 & 0.0009835 & 0.03701 & 0.008824 & 0.009102 & 0.3082 & & 0.5762 & 0.4899 & 1 & 0.02048 & 0.3641 \\
\hline SNP3 & rs1165687 & 0.2644 & 0.3561 & 0.3567 & 0.361 & 0.06431 & 0.03972 & & 1 & 1 & 1 & 0.9739 \\
\hline SNP4 & rs1882019 & 0.02699 & 0.05905 & 0.04886 & 0.04939 & 0.723 & 0.2152 & 0.1073 & & 1 & 1 & 1 \\
\hline SNP5 & rs17034977 & 0.009254 & 0.01494 & 0.08256 & 0.08151 & 0.01216 & 0.01078 & 0.0788 & 0.009672 & & 1 & 1 \\
\hline SNP6 & rs703657 & 0.511 & 0.1602 & 0.3653 & 0.3716 & 0.04883 & $2.69 \mathrm{E}-05$ & 0.469 & 0.05756 & 0.04227 & & 1 \\
\hline SNP7 & rs2293618 & 0.03986 & 0.07896 & 0.001341 & 0.001146 & 0.346 & 0.07587 & 0.1983 & 0.5131 & 0.3768 & 0.1122 & \\
\hline
\end{tabular}

Fig. 3 Intermarker linkage disequilibrium pattern in NIMH trio samples. The standardized disequilibrium coefficient $\left(D^{\prime}\right)$ and squared correlation coefficient $\left(r^{2}\right)$ was calculated for transmitted alleles using the TDTPHASE program. $D^{\prime}$ larger than 0.9 are shown in shaded cells 


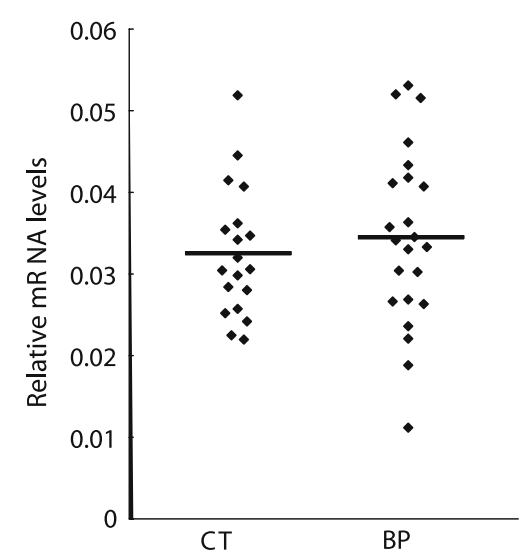

Fig. 4 HSP9OB1 mRNA levels in lymphoblastoid cells. $Y$-axis indicates the relative ratio of $H S P 90 B 1$ to GAPDH. No significant difference was observed between the cases of bipolar disorder (BP) and controls (CT)

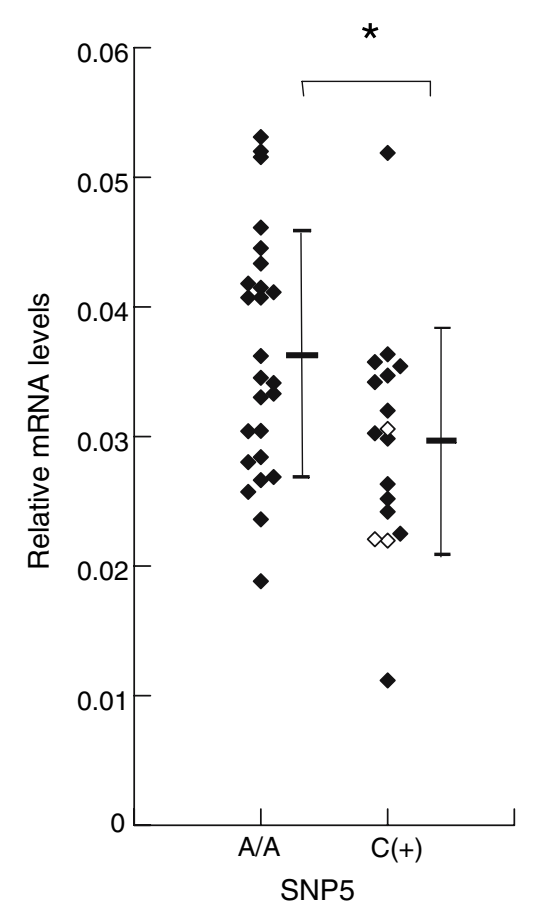

Fig. 5 Relationship between HSP90B1 mRNA levels and SNP5 (rs17034977) in lymphoblastoid cells. $Y$-axis indicates the relative ratio of $H S P 90 B 1$ to $G A P D H$. A statistically significant difference of HSP90B1 levels was observed between the homozygous A allele carriers and the $\mathrm{C}$ allele carriers. Open square indicates homozygote for the C allele of SNP $5(n=3)$. The bars show the average and standard deviations. ${ }^{*} P=0.035$, Mann-Whitney $U$ test

is a conserved sequence in the upstream region of HSP90B1, corresponding to the common virus integration site Evi12, the target of Cas-Br-M murine leukemia virus in mice (van den Akker et al. 2005). In the case of mice, integration of retrovirus was postulated to affect the expression level of HSP9OB1 (Valk et al. 1999; van den Akker et al. 2005). There has been no evidence that supports the integration of retrovirus in this site in humans, and the present results also excluded such a possibility. Although the results of haplotype analysis, association analysis, mutation search, and mRNA analysis suggest that SNP5 itself might be important, it is still not known whether SNP5 itself affects the mRNA levels. Further studies will be needed to identify the genetic polymorphism affecting the mRNA levels.

Stratification analysis in Japanese samples including the part of the present samples did not show evidence suggesting the existence of population stratification (Yamada et al. 2004). Thus, it is unlikely that the observed difference is the artifact due to population stratification. However, it is possible that some control subjects might actually have bipolar disorder but have reported themselves as healthy, because patients with bipolar disorder often do not have insight into the illness. This might have confounded the results.

Lack of association in NIMH samples might suggest that the association between the SNP5 and bipolar disorder is ethnicity dependent. Indeed, it was reported that many associations between SNPs and expression levels were ethnicity dependent (Stranger et al. 2007).

A possibility that SNP5 is also associated with schizophrenia cannot be ruled out, because a similar tendency of association was observed $(P=0.09)$. This should be tested in larger samples.

Although the C allele of SNP5, a protective SNP, was associated with significantly lower mRNA levels of $H S P 90 B 1$, the HSP90B1 mRNA levels in lymphoblastoid cells did not show a significant difference between controls and patients. Although the possible effects of medication cannot be totally ruled out, such effect should be minimized because we cultured the lymphoblastoid cells at least for 1 month in culture media free from psychotropic drugs. It is not known how lower expression leves of HSP90B1 reduce a risk for bipolar disorder in Japanese. HSP90B1, or GRP94, is an ER chaperone which binds to various proteins and peptides in a non-specific manner (Argon and Simen 1999). It also serves as a major $\mathrm{Ca}^{2+}$ buffer of the ER (Nigam et al. 1994). Overexpression of GRP94 is reported to protect the cells from death (Bando et al. 2004). Thus, the protective effect of the allele reducing the expression level of HSP90B1 against bipolar disorder is apparently contradictory to a hypotheseis that vulnerability to cell death may play a role in the pathophysiology of bipolar disorder (Manji and Duman 2001). However, it is not known whether gene expression in lymphoblastoid cells reflects the alteration in the brain. Transcriptional regulation may differ between lymphoblastoid cells and neuronal cells. Thus, it is too premature to assume that subjects carrying SNP5 have lower expression levels of HSP90B1 in neuronal cells. 
In conclusion, we found an association of rs17034977 in HSP90B 1 with bipolar disorder in a Japanese population and suggested that the alteration of mRNA expression by this SNP could be an underlying mechanism for bipolar disorder.

Acknowledgments This research was supported by a grant for Laboratory for Molecular Dynamics of Mental Disorders, RIKEN Brain Science Institute, a Grant-in-Aid from Japanese Ministry of Health and Labor, and a Grant-in-Aid from the Japanese Ministry of Education, Culture, Sports, Science and Technology. The authors declare no conflict of interest.

\section{References}

Argon Y, Simen BB (1999) GRP94, an ER chaperone with protein and peptide binding properties. Semin Cell Dev Biol 10:495-505

Arinami T, Ohtsuki T, Ishiguro H, Ujike H, Tanaka Y, Morita Y et al (2005) Genomewide high-density SNP linkage analysis of 236 Japanese families supports the existence of schizophrenia susceptibility loci on chromosomes 1p, 14q, and 20p. Am J Hum Genet 77:937-944

Bando Y, Katayama T, Aleshin AN, Manabe T, Tohyama M (2004) GRP94 reduces cell death in SH-SY5Y cells perturbated calcium homeostasis. Apoptosis 9:501-508

Bown C, Wang JF, MacQueen G, Young LT (2000) Increased temporal cortex ER stress proteins in depressed subjects who died by suicide. Neuropsychopharmacology 22:327-332

Cichon S, Buervenich S, Kirov G, Akula N, Dimitrova A, Green E et al (2004) Lack of support for a genetic association of the XBP1 promoter polymorphism with bipolar disorder in probands of European origin. Nat Genet 36:783-784, author reply 784785

Curtis D, Kalsi G, Brynjolfsson J, McInnis M, O’Neill J, Smyth C et al (2003) Genome scan of pedigrees multiply affected with bipolar disorder provides further support for the presence of a susceptibility locus on chromosome $12 \mathrm{q} 23-\mathrm{q} 24$, and suggests the presence of additional loci on 1p and 1q. Psychiatr Genet 13:7784

Dawson E, Parfitt E, Roberts Q, Daniels J, Lim L, Sham P et al (1995) Linkage studies of bipolar disorder in the region of the Darier's disease gene on chromosome 12q23-24.1. Am J Med Genet 60:94-102

Detera-Wadleigh SD, Badner JA, Berrettini WH, Yoshikawa T, Goldin LR, Turner G et al (1999) A high-density genome scan detects evidence for a bipolar-disorder susceptibility locus on $13 \mathrm{q} 32$ and other potential loci on 1q32 and 18p11.2. Proc Natl Acad Sci USA 96:5604-5609

Dow GS, Hudson TH, Vahey M, Koenig ML (2003) The acute neurotoxicity of mefloquine may be mediated through a disruption of calcium homeostasis and ER function in vitro. Malar J 2:14

Dow GS, Caridha D, Goldberg M, Wolf L, Koenig ML, Yourick DL, Wang Z (2005) Transcriptional profiling of mefloquine-induced disruption of calcium homeostasis in neurons in vitro. Genomics 86:539-550

Ekholm JM, Kieseppa T, Hiekkalinna T, Partonen T, Paunio T, Perola $\mathrm{M}$ et al (2003) Evidence of susceptibility loci on 4q32 and 16p12 for bipolar disorder. Hum Mol Genet 12:1907-1915

Even C, Friedman S, Lanouar K (2001) Bipolar disorder after mefloquine treatment. J Psychiatry Neurosci 26:252-253

Ewald H, Degn B, Mors O, Kruse TA (1998) Significant linkage between bipolar affective disorder and chromosome 12q24. Psychiatr Genet 8:131-140
Green E, Elvidge G, Jacobsen N, Glaser B, Jones I, O’Donovan MC et al (2005) Localization of bipolar susceptibility locus by molecular genetic analysis of the chromosome 12q23-q24 region in two pedigrees with bipolar disorder and Darier's disease. Am J Psychiatry 162:35-42

Hiroi T, Wei H, Hough C, Leeds P, Chuang DM (2005) Protracted lithium treatment protects against the ER stress elicited by thapsigargin in rat PC12 cells: roles of intracellular calcium, GRP78 and Bcl-2. Pharmacogenomics J 5:102-111

Hou SJ, Yen FC, Cheng CY, Tsai SJ, Hong CJ (2004) X-box binding protein 1 (XBP1) C-116G polymorphisms in bipolar disorders and age of onset. Neurosci Lett 367:232-234

Jayanthi S, Deng X, Noailles PA, Ladenheim B, Cadet JL (2004) Methamphetamine induces neuronal apoptosis via cross-talks between endoplasmic reticulum and mitochondria-dependent death cascades. Faseb J 18:238-251

Jurata LW, Bukhman YV, Charles V, Capriglione F, Bullard J, Lemire AL et al (2004) Comparison of microarray-based mRNA profiling technologies for identification of psychiatric disease and drug signatures. J Neurosci Methods 138:173-188

Kakiuchi C, Iwamoto K, Ishiwata M, Bundo M, Kasahara T, Kusumi I et al (2003) Impaired feedback regulation of XBP1 as a genetic risk factor for bipolar disorder. Nat Genet 35:171-175

Kakiuchi C, Ishiwata M, Nanko S, Kunugi H, Minabe Y, Nakamura K et al (2005) Functional polymorphisms of HSPA5: possible association with bipolar disorder. Biochem Biophys Res Commun 336:1136-1143

Kakiuchi C, Ishiwata M, Hayashi A, Kato T (2006) XBP1 induces WFS1 through an endoplasmic reticulum stress response element-like motif in SH-SY5Y cells. J Neurochem 97:545-555

Kakiuchi C, Ishiwata M, Nanko S, Kunugi H, Minabe Y, Nakamura K et al (2007) Association analysis of ATF4 and ATF5, genes for interacting-proteins of DISC1, in bipolar disorder. Neurosci Lett 417:316-321

Kato T (2007) Molecular genetics of bipolar disorder and depression. Psychiatry Clin Neurosci 61:3-19

Kato T, Ishiwata M, Mori $\mathrm{K}$, Washizuka S, Tajima O, Akiyama T, Kato N (2003) Mechanisms of altered $\mathrm{Ca}^{2+}$ signalling in transformed lymphoblastoid cells from patients with bipolar disorder. Int J Neuropsychopharmacol 6:379-389

Kim AJ, Shi Y, Austin RC, Werstuck GH (2005) Valproate protects cells from ER stress-induced lipid accumulation and apoptosis by inhibiting glycogen synthase kinase-3. J Cell Sci 118:89-99

Manji HK, Duman RS (2001) Impairments of neuroplasticity and cellular resilience in severe mood disorders: implications for the development of novel therapeutics. Psychopharmacol Bull 35:549

Maziade M, Roy MA, Chagnon YC, Cliche D, Fournier JP, Montgrain N et al (2005) Shared and specific susceptibility loci for schizophrenia and bipolar disorder: a dense genome scan in Eastern Quebec families. Mol Psychiatry 10:486-499

Morissette J, Villeneuve A, Bordeleau L, Rochette D, Laberge C, Gagne B et al (1999) Genome-wide search for linkage of bipolar affective disorders in a very large pedigree derived from a homogeneous population in quebec points to a locus of major effect on chromosome 12q23-q24. Am J Med Genet 88:567-587

Nigam SK, Goldberg AL, Ho S, Rohde MF, Bush KT, Sherman M (1994) A set of endoplasmic reticulum proteins possessing properties of molecular chaperones includes $\mathrm{Ca}(2+)$-binding proteins and members of the thioredoxin superfamily. J Biol Chem 269:1744-1749

Schroder M, Kaufman RJ (2005) ER stress and the unfolded protein response. Mutat Res 569:29-63

Shao L, Sun X, Xu L, Young LT, Wang JF (2006) Mood stabilizing drug lithium increases expression of endoplasmic reticulum 
stress proteins in primary cultured rat cerebral cortical cells. Life Sci 78:1317-1323

Sheehan DV, Lecrubier Y, Sheehan KH, Amorim P, Janavs J, Weiller E et al (1998) The Mini-International Neuropsychiatric Interview (M.I.N.I.): the development and validation of a structured diagnostic psychiatric interview for DSM-IV and ICD-10. J Clin Psychiatry 59(Suppl 20):22-33, quiz 34-57

So J, Warsh JJ, Li PP (2007) Impaired endoplasmic reticulum stress response in B-lymphoblasts from patients with bipolar-I disorder. Biol Psychiatry 62:141-147

Stranger BE, Forrest MS, Dunning M, Ingle CE, Beazley C, Thorne N et al (2007) Relative impact of nucleotide and copy number variation on gene expression phenotypes. Science 315:848-853

Toda H, Suzuki G, Nibuya M, Shioda K, Nishijima K, Wakizono T et al (2006) Behavioral stress and activated serotonergic neurotransmission induce XBP-1 splicing in the rat brain. Brain Res 1112:26-32

Valk PJ, Vankan Y, Joosten M, Jenkins NA, Copeland NG, Lowenberg B, Delwel R (1999) Retroviral insertions in Evi12, a novel common virus integration site upstream of Tra1/Grp94, frequently coincide with insertions in the gene encoding the peripheral cannabinoid receptor Cnr2. J Virol 73:3595-3602

van den Akker E, Vankan-Berkhoudt Y, Valk PJ, Lowenberg B, Delwel R (2005) The common viral insertion site Evi12 is located in the 5'-noncoding region of Gnn, a novel gene with enhanced expression in two subclasses of human acute myeloid leukemia. J Virol 79:5249-5258

Wang JF, Bown C, Young LT (1999) Differential display PCR reveals novel targets for the mood-stabilizing drug valproate including the molecular chaperone GRP78. Mol Pharmacol 55:521-527

Wang JF, Azzam JE, Young LT (2003) Valproate inhibits oxidative damage to lipid and protein in primary cultured rat cerebrocortical cells. Neuroscience 116:485-489

Yamada K, Nakamura K, Minabe Y, Iwayama-Shigeno Y, Takao H, Toyota $\mathrm{T}$ et al (2004) Association analysis of FEZ1 variants with schizophrenia in Japanese cohorts. Biol Psychiatry 56:683-690

Yoshida H (2004) Molecular biology of the ER stress response. Seikagaku 76:617-630 